\title{
Human neural stem cell growth and differentiation in a gradient-generating microfluidic device $\dagger$
}

\author{
Bong Geun Chung,,$^{a}$ Lisa A. Flanagan, $\dagger^{b}$ Seog Woo Rhee, ${ }^{a}$ Philip H. Schwartz, ${ }^{c d}$ Abraham P. Lee, ${ }^{a}$ \\ Edwin S. Monuki*bc and Noo Li Jeon*a
}

Received 22nd November 2004, Accepted 17th February 2005

First published as an Advance Article on the web 9th March 2005

DOI: $10.1039 / \mathrm{b} 417651 \mathrm{k}$

This paper describes a gradient-generating microfluidic platform for optimizing proliferation and differentiation of neural stem cells (NSCs) in culture. Microfluidic technology has great potential to improve stem cell (SC) cultures, whose promise in cell-based therapies is limited by the inability to precisely control their behavior in culture. Compared to traditional culture tools, microfluidic platforms should provide much greater control over cell microenvironment and rapid optimization of media composition using relatively small numbers of cells. Our platform exposes cells to a concentration gradient of growth factors under continuous flow, thus minimizing autocrine and paracrine signaling. Human NSCs (hNSCs) from the developing cerebral cortex were cultured for more than 1 week in the microfluidic device while constantly exposed to a continuous gradient of a growth factor (GF) mixture containing epidermal growth factor (EGF), fibroblast growth factor 2 (FGF2) and platelet-derived growth factor (PDGF). Proliferation and differentiation of NSCs into astrocytes were monitored by time-lapse microscopy and immunocytochemistry. The NSCs remained healthy throughout the entire culture period, and importantly, proliferated and differentiated in a graded and proportional fashion that varied directly with GF concentration. These concentration-dependent cellular responses were quantitatively similar to those measured in control chambers built into the device and in parallel cultures using traditional 6-well plates. This gradient-generating microfluidic platform should be useful for a wide range of basic and applied studies on cultured cells, including SCs.

\section{Introduction}

Stem cells (SCs) hold tremendous promise for fundamental biological studies and cell-based therapies in human disorders. ${ }^{1-4}$ Among other advantages, SCs can be expanded in culture, genetically modified using existing gene delivery tools, and differentiated into cell types needed for cell replacement or tissue engineering. ${ }^{3-5}$ SCs therefore provide a multipronged approach to potential cell-based therapies. ${ }^{3,4,6}$

The promise of SCs holds true for tissue-specific SCs as well, such as neural stem cells (NSCs). ${ }^{5}$ NSCs are defined as cells that can self-renew yet maintain the ability to generate the three principal cell types of the central nervous system (CNS) - neurons, astrocytes, and oligodendrocytes. ${ }^{6-8}$ NSCs therefore have therapeutic possibilities in multiple neurodevelopmental and neurodegenerative diseases, such as Alzheimer's and Parkinson's diseases, ${ }^{3,4,9}$ for which effective therapies are limited or lacking.

Unfortunately, several concerns with SC cultures currently limit their therapeutic promise. ${ }^{3}$ One major problem is the inability to precisely control SC behavior in culture, such as their proliferation and specification into different cell

$\uparrow$ Electronic supplementary information (ESI) available: Time lapse movie of gradient chamber. Fig. S1, Tables S1 and S2. See http:// www.rsc.org/suppdata/lc/b4/b417651k/

\$ These two authors contributed equally to this work.

*emonuki@uci.edu (Edwin S. Monuki)

njeon@uci.edu (Noo Li Jeon) types. ${ }^{10,11}$ Conventional cell culture technology is ill-suited to tackle this problem. For example, precise and thorough combinatorial studies with multiple growth factors are technically infeasible using traditional cultures, and traditional cultures require relatively large volumes of media and correspondingly large amounts of costly growth factors and other reagents. ${ }^{12,13}$

Microfluidic cell cultures have the potential to overcome some of the problems imposed by traditional culture tools. ${ }^{12,13}$ Advances in soft lithography and microfluidics have enabled the development of microfluidic devices for cell-based applications. ${ }^{12,14}$ We have previously reported microfluidic chemotaxis chambers ${ }^{15,16}$ that can generate precise concentration mixtures and allow real-time observation of migrating cells. This approach was used to investigate chemotaxis of neutrophils in chemokine (Interleukin-8) gradients ${ }^{15}$ and metastatic breast cancer cells in epidermal growth factor (EGF) gradients. ${ }^{16}$ Due to their biocompatibility, poly(dimethylsiloxane) (PDMS) microfluidic devices are increasingly used as microscale incubators to culture a variety of cells for days to weeks. ${ }^{17-20}$

This paper describes an experimental platform for optimizing proliferation and differentiation conditions for NSCs using a gradient-generating microfluidic device. ${ }^{21,22}$ In this device, we cultured human NSCs (hNSCs) isolated from the developing cerebral cortex in a continuous concentration gradient of known growth factors. These cells exhibited proliferation and differentiation responses that were concentration-dependent and quantitatively similar to those seen in parallel control cultures. 


\section{Experimental}

\section{Fabrication of the microfluidic device}

The microfluidic NSC culture device was fabricated in PDMS using rapid prototyping and soft lithography following published procedures. ${ }^{15,21,23-26}$ The master mold was fabricated by patterning $100 \mu \mathrm{m}$ thick negative photoresist (SU8 50, Microchem, MA) by photolithography. ${ }^{22}$ A positive replica with an embossed network of microchannels was fabricated by replica molding PDMS against the master. ${ }^{21,23-25}$ The surface of PDMS and glass slide were activated with reactive oxygen plasma (2 min at 30W, model PDC-001, Harrick Scientific, NY) and brought together immediately to form an irreversible seal. $^{15,22}$ The PDMS was attached to the glass slide such that glass forms the bottom of the channels and provides a surface for cellular adhesion. The cell culture area (near the outlet, $2.4 \mathrm{~mm}$ wide channel in gradient devices) was covered with a glass slide during plasma treatment to prevent permanent bonding. This step allowed removal of PDMS portion around the cell culture area for immunocytochemistry and fluorescence microscopy. After assembly, the device surface was coated with poly-L-lysine (PLL; $0.5 \mathrm{mg} \mathrm{mL}^{-1}$ in $0.1 \mathrm{M}$ borate buffer at room temperature for $1 \mathrm{~h})^{30,31}$ and mouse laminin $\left(0.5 \mathrm{mg} \mathrm{mL}{ }^{-1}\right.$ at $37{ }^{\circ} \mathrm{C}$ for $\left.8 \mathrm{~h}\right)$. Microfluidic devices were connected to syringe pumps with base medium (left 2 inlets) or base medium with growth factors (right inlet) to generate a spatial concentration gradient (combinatorial mixture) of the growth factors perpendicular to the channel across the cell culture area. ${ }^{15,22}$ The stability and shape of the soluble growth factor gradient was confirmed by imaging fluorescence from fluorescein isothiocyanate (FITC)-dextran. FITC-dextran (10 $\mu \mathrm{M}, \mathrm{MW} 10,000)$, which has a similar molecular weight to growth factors like EGF (MW 6,000), was added to the growth factor-containing media to serve as an indirect indicator of the growth factor gradient inside the channel. ${ }^{16}$ The observed gradient profile matched the mathematical prediction as in previous work. ${ }^{21,22}$

\section{Human NSC (hNSC)}

The hNSCs isolated from the developing cerebral cortex of premature human infants ${ }^{1,2}$ (SC23) were provided by the National Human Neural Stem Cell Resource at the Children's Hospital of Orange County, and grown as adherent cultures in fibronectin-coated flasks in a humidified incubator at $37{ }^{\circ} \mathrm{C}$ with $5 \% \mathrm{CO}_{2}$. Cells were cultured in base medium consisting of Dulbecco's modified Eagle's medium: F12 nutrient mixtures (DMEM:F12, Invitrogen, CA) supplemented with 20\% BIT 9500 (Stem Cell Technologies, Canada) and 1\% antibiotic/ antimycotic (Invitrogen, CA). ${ }^{1,2}$ Growth medium was prepared from base medium by adding three human recombinant growth factors, each at $40 \mathrm{ng} \mathrm{mL}^{-1}$ : epidermal growth factor (EGF, BD Biosciences, MA), fibroblast growth factor 2 (FGF2, BD Biosciences, MA), and platelet-derived growth factor (PDGF, PeproTech, NJ).,

\section{Microfluidic cultures and time-lapse microscopy}

The hNSCs were detached from the culture flask using Cell Dissociation Buffer (Invitrogen, CA), washed, and resuspended in culture medium. Cells $(100 \mu \mathrm{L}$ suspension of 500,000 cells $\mathrm{mL}^{-1}$ ) were loaded into the microfluidic device via cell inlet ports on sides of the chambers using a micropipette tip. The microfluidic device containing adherent hNSCs was placed inside an environmental chamber $\left(37{ }^{\circ} \mathrm{C}\right.$ and $5 \% \mathrm{CO}_{2}$ ) on an inverted microscope (Nikon, NY) (see ESI Fig. $\mathrm{S} 1 \uparrow$ for a picture of the experimental setup). The microfluidic chamber was maintained with a constant perfusion of media at physiologic $\mathrm{pH}$. Polyethylene tubing was inserted into three inlets at the top of the gradient chamber to make fluidic connections. ${ }^{15}$ The media with and without GF were injected into the gradient chamber using a syringe pump at a controlled rate of $0.1 \mu \mathrm{L} \min ^{-1}$. Cells cultured inside microfluidic gradient chambers were thus exposed to a GF gradient in a stable microenvironment. A motorized stage was used to obtain images at multiple positions in the microfluidic device at $10 \mathrm{~min}$ intervals.

\section{Immunocytochemistry}

After culturing the cells inside microfluidic devices for 7 days, they were fixed in 4\% paraformaldehyde (Sigma, MO) and permeabilized with $0.3 \%$ Triton X-100 in phosphate buffered saline (PBS, Invitrogen, CA). After washing with PBS, cells were blocked with $5 \%$ bovine serum albumin (BSA, Sigma, MO) in PBS and sequentially exposed to primary and secondary antibodies: anti-glial fibrillary acidic protein (GFAP, Chemicon International, CA; overnight at $4{ }^{\circ} \mathrm{C}$ ) and FITC-conjugated AffiniPure donkey anti-rabbit immunoglobulin $\mathrm{G}$ (IgG) (Jackson ImmunoResearch Laboratories Inc, PA; $2 \mathrm{~h}$ at room temperature). Cell nuclei were then counterstained with Hoechst 33342 (Molecular Probes, OR) and devices mounted with Vectashield mounting medium (Vector Laboratories Inc, CA). Immunostained cells were kept at $4{ }^{\circ} \mathrm{C}$ until analyzed. Fluorescent images were obtained with a Nikon E800 upright microscope using a chroma standard filter $(350 \mathrm{~nm})$ for DAPI/Hoechst and a chroma high $\mathrm{Q}$ filter $(480 \mathrm{~nm})$ for FITC.

\section{Results and discussion}

\section{Design and validation of the gradient-generating device}

The device is made up of two types of chambers, a gradient chamber and two control chambers. The first chamber is composed of two parts: (1) the gradient-generating microchannel network ${ }^{15}$ ("Christmas tree"), which utilizes laminar flow and diffusive mixing to generate a continuous combinatorial mixture of soluble factors ${ }^{20,26,29}$ and (2) the cell culture area near the bottom of the device across which the gradient is applied. Two control cell culture chambers are also fabricated next to the main gradient chamber for positive and negative controls $(100 \%+\mathrm{GF}$ and $-\mathrm{GF})$.

The gradient chamber network is supplied with media via three separate inlets from the top of the device (Fig. 1A). The shape of gradient produced can be controlled by adjusting the concentration of solutions for the three inlets. ${ }^{15,21,22}$ For this experiment, we generated a polynomial gradient $\left(y=a x^{2}+b\right)$ by loading the first two inlets with media lacking growth factors $(0 \%, "-G F "$ in Fig. 1A) and the third inlet 


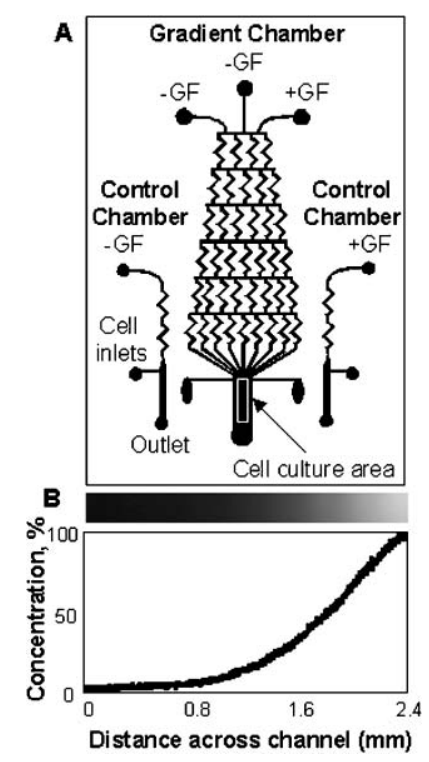

Fig. 1 (A) Schematic design of the microfluidic device showing the gradient chamber and two control chambers. Cells were loaded into the chambers via inlet ports, and the entire device was placed on an inverted microscope for live-cell imaging. (B) The gradient profile generated within the gradient chamber was visualized with the fluorescence profile of FITC-dextran.

with media containing the full complement of GFs $(100 \%$, "+GF" in Fig. 1A). ${ }^{22,32}$ In order to confirm the generation of a gradient with this device, we placed a solution of FITC-dextran in the third inlet and solutions without FITC-dextran in the first two inlets. Using computer controlled-syringe pumps, we were able to generate a gradient of fluorescence with micron-scale spatial resolution across the chamber (Fig. 1B). The GFs (EGF, FGF2, PDGF) used have similar molecular weights to FITC-dextran, making it likely that the GF gradient is the same as the observed fluorescence gradient from FITC-dextran (Fig. 1B).

\section{Human NSCs in control chambers}

The control chambers are $500 \mu \mathrm{m}$ wide and $100 \mu \mathrm{m}$ high; the gradient chamber is $2.4 \mathrm{~mm}$ wide and $100 \mu \mathrm{m}$ high with two barriers at $0.8 \mathrm{~mm}$ intervals to limit cell migration across the width of the chamber. Both the control and gradient chambers have outlet ports for fluid exit. Since fluid is pumped into the device at a controlled rate of $0.1 \mu \mathrm{L} \mathrm{min}{ }^{-1}$, the cells in the gradient chamber would be theoretically subjected to minimal fluid shear stress on the order of $5 \times 10^{-4}$ dyne $\mathrm{cm}^{-2}$ (channel dimension of $2.4 \mathrm{~mm} \times 10 \mathrm{~mm} \times 0.1 \mathrm{~mm}$ ). This is significantly lower than physiological shear stress experienced by endothelial cells during normal blood flow $\left(\sim 2\right.$ dyne $\left.\mathrm{cm}^{-2}\right){ }^{33}$ In addition, smaller volumes of solutions were sufficient for long term experiments compared to conventional methods ( $\sim 1 \mathrm{~mL}$ solution per syringe was adequate for a week-long experiment with constant perfusion).

We then tested whether hNSCs could be successfully cultured in the microfluidic device under continuous fluid flow. The chambers were first precoated with PLL/laminin to encourage cell adhesion. Dissociated hNSCs were plated into control chambers at a density of $5 \times 10^{5}$ cells $\mathrm{mL}^{-1}$, allowed to adhere, then exposed to media with or without EGF, FGF2, and PDGF. ${ }^{1,2}$ These cells are known to proliferate in the presence of these GFs and differentiate in their absence. ${ }^{2,27,28}$ Images taken immediately after plating show that the cells were well adhered and evenly distributed across the chambers, but not yet elongated (Fig. 2A, C). After 7 days, cells in media lacking GFs were elongated and had extended processes, but showed little proliferation, with comparable cell numbers at day 7 compared to day 1 (Fig. 2B). Cells in media with GFs were also elongated after 7 days, but showed a striking increase in cell number (Fig. 2D). Cell numbers in the control chambers under flow were occasionally lower than those in conventional culture dishes lacking fluid flow (Fig. 2E, F). This would be consistent with the removal of proliferative autocrine and paracrine factors in the control microfluidic chambers, but not in the traditional cultures. These studies showed that hNSCs could be loaded evenly in the chamber, adhered and elongated along the surface, remained healthy for at least one week under fluidic flow, and proliferated in response to mitogenic GFs. Moreover, the cells in microfluidic chambers behaved similarly to those in conventional culture dishes.

\section{Human NSC proliferation in the gradient chamber}

We then cultured cells in the gradient chamber of the microfluidic device. The gradient chamber had two barriers that limited cell migration and divided the continuous GF gradient into three compartments with low, intermediate, or high concentrations of GFs; $0-2.8 \mathrm{ng} \mathrm{mL}^{-1}(0-7 \%), 3.2-14 \mathrm{ng} \mathrm{mL}^{-1}$ (8-35\%), and 14.4-40 $\mathrm{ng} \mathrm{mL}^{-1}(36-100 \%)$ for each GF.
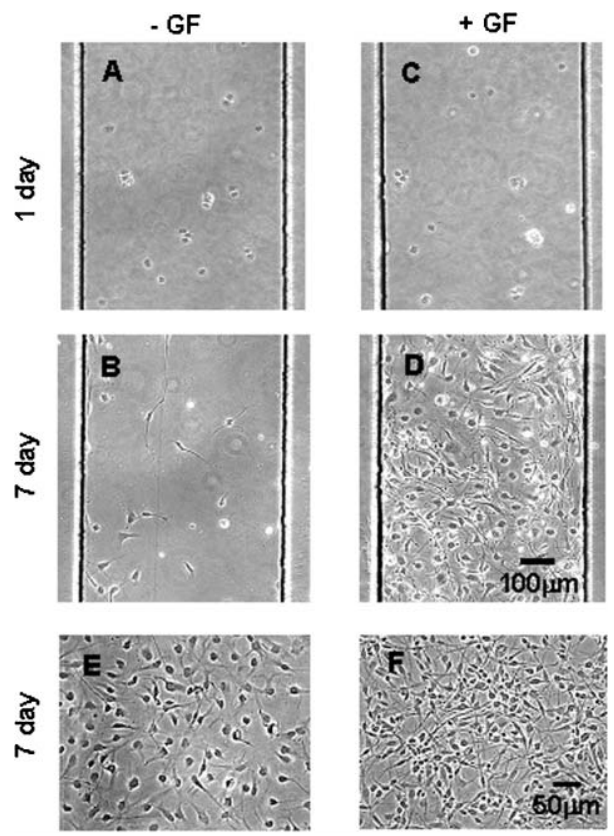

Fig. 2 Proliferation of hNSCs in control microfluidic chambers. Phase contrast images show cells in control chambers (A-D) or on traditional coverslips (E, F). Cells in the chambers were grown in fluid flow conditions using media with $(+\mathrm{GF})$ or without $(-\mathrm{GF})$ growth factors. Cells on coverslips in conventional cultures were grown in the same media for 7 days. 
Soon after plating (on day 1), the cells had adhered to the glass surface, but were fairly sparse in all three compartments (Fig. 3A). After 4 days, the cells had elongated and undergone limited proliferation in the low GF compartment, some proliferation in the intermediate GF compartment, and more proliferation in the high GF compartment (Fig. 3B). This trend continued through day 7, when many more cells were evident in the high GF compartment (see ESI $\dagger$ for time-lapse movies). In addition to the effects on cell proliferation, the GF gradient within the intermediate and high GF compartments stimulated cell migration towards the higher GF concentrations (e.g. Fig. 3B and time-lapse movies in ESI $\dagger$ ).

Quantification of total cell numbers revealed that the hNSCs proliferated in direct proportion to the GF concentration. The hNSCs plated in the control chamber without GFs showed no cell number increase at 4 or 7 days (Fig. 4A). However, cells in the low GF compartment of the gradient chamber (0-2.8 ng mL ${ }^{-1}$ each GF) showed a 1.8-fold increase at 4 days and a 2.6-fold increase by day 7 (Fig. 4B). Cells in the

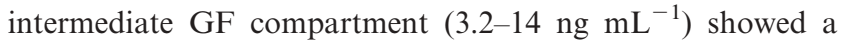
2.5-fold increase on day 4 and 3.5-fold increase on day 7, while cell numbers in the high GF compartment (14.4-40 $\mathrm{ng} \mathrm{mL}^{-1}$ ) increased by 3.5 -fold and 5-fold at 4 and 7 days, respectively (Fig. 4B). The control chamber with $100 \%$ GF concentration showed increases of 4 -fold on day 4 and 6.8 -fold on day 7 (Fig. 4A). We then subdivided each compartment arbitrarily into 8 equal bins $(100 \mu \mathrm{m}$ wide, Fig. 3B) and reanalyzed the data. As shown in Fig. 4C, we continued to find a strong correlation between cell number and GF concentration in all three compartments (Fig. 4C and data not shown; ESI Table $\mathrm{S} 1 \uparrow$ for $p$ values), despite the cell migration observed. Therefore, by culturing hNSCs under a continuous gradient of
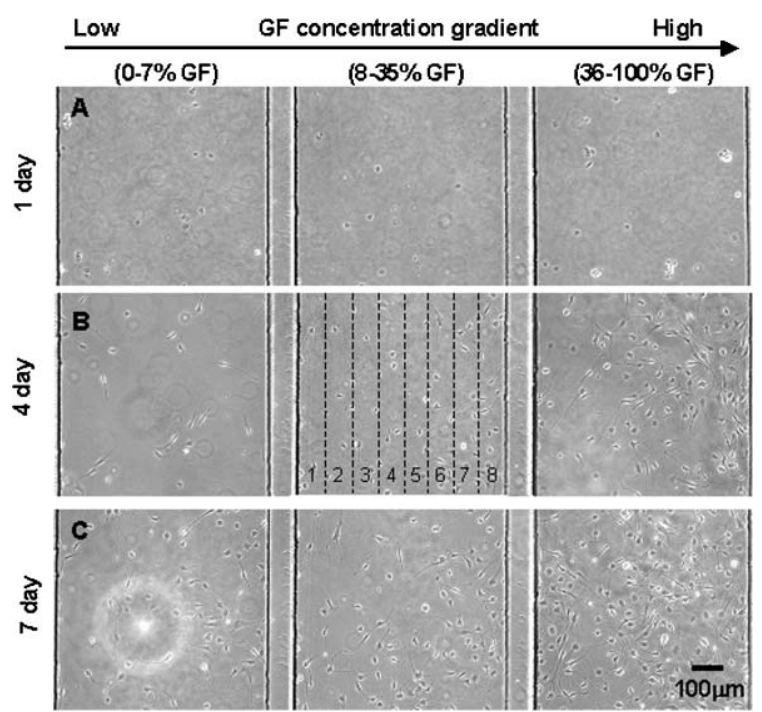

Fig. 3 Proliferation of hNSCs in the gradient chamber. Phase contrast images show cells cultured for up to 7 days in the gradient chamber. The concentration gradient is continuous across the entire chamber, which is divided into three compartments by physical barriers to limit migration. By the end of the culture period, cell numbers were greatest in the high GF compartment (36-100\% GF) and lowest in the low GF compartment (0-7\% GF). Dashed lines delineate the 8 equal bins used for quantification in Figs. $4 \mathrm{C}$ and $6 \mathrm{C}$.
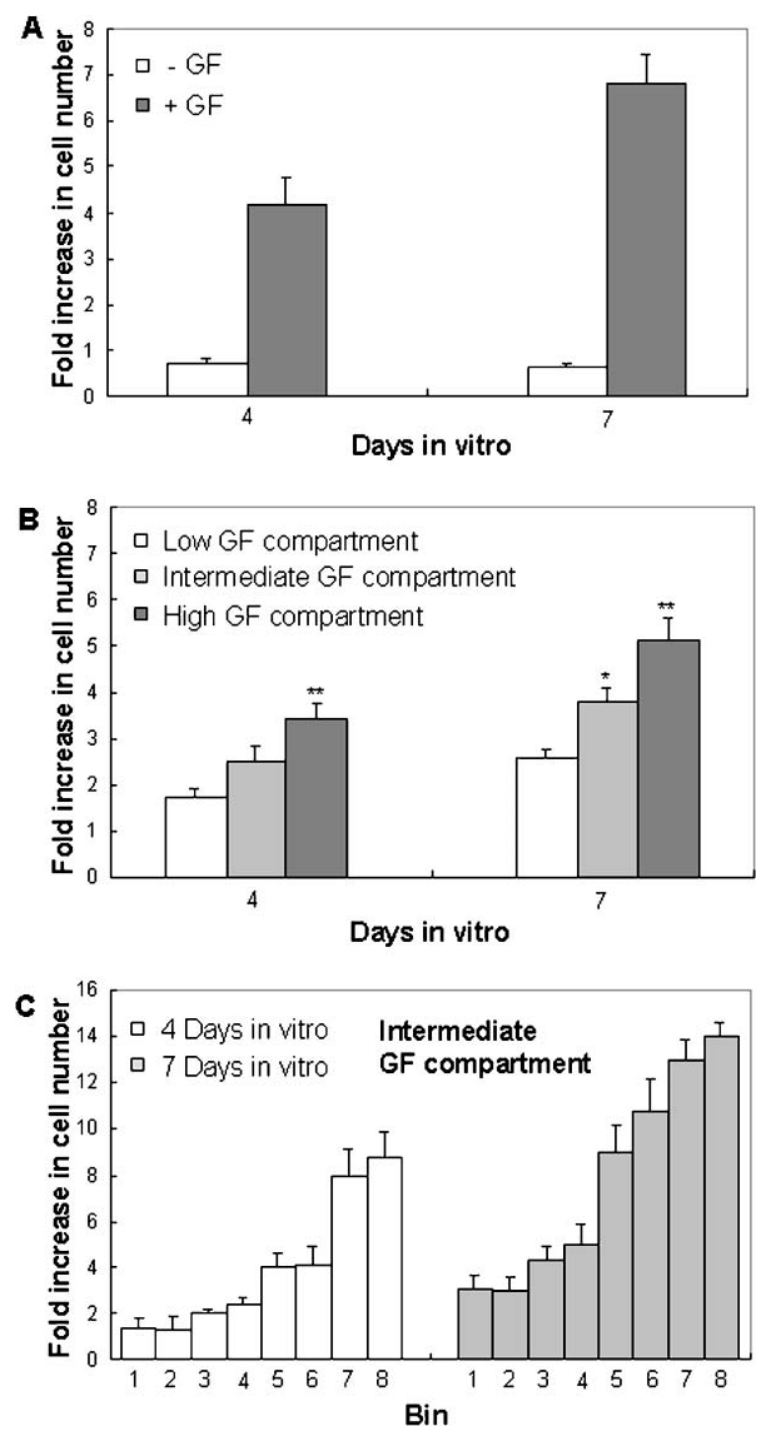

Fig. 4 Quantitative analysis of hNSC proliferation in the control (A) or gradient chambers (B, C) at 4 and 7 days. The three compartments of the gradient chamber are termed low, intermediate, and high. The fold increase in cell number represents cell numbers on day 4 or 7 divided by initial cell number on day 1 . Note that cell numbers are directly proportional to GF concentration in each gradient compartment. Each bar represents the average for 3 independent experiments with standard errors of the mean $\left({ }^{*} p<0.05,{ }^{* *} p<0.01\right)$. Quantitation within the subdivided intermediate GF compartment shows a strong positive correlation between cell number and GF concentration (C; ESI Table $\mathrm{S} 1 \uparrow$ for $p$ values).

GFs, we showed that these cells respond in direct proportion to GF concentration, at least within the $0-40 \mathrm{ng} \mathrm{mL}^{-1}$ range for EGF, FGF2 and PDGF, and that there is no threshold or "all-or-none" proliferation response in this concentration range.

\section{Differentiation of human NSCs into astrocytes in the gradient chamber}

Since GF concentration directed hNSC proliferation, we tested whether the GF gradient also regulated their differentiation. ${ }^{2}$ Cells grown for one week in the GF gradient were fixed and 

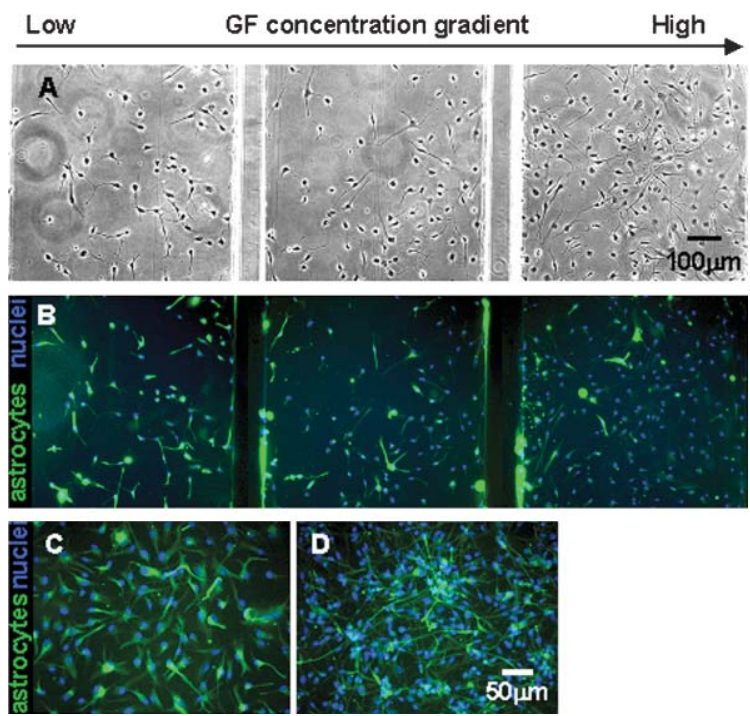

Fig. 5 Differentiation of hNSCs into astrocytes in the gradient chamber. The hNSCs cultured in the gradient chamber $(\mathrm{A}, \mathrm{B})$ or on coverslips $(0 \% \mathrm{GF}$ in $\mathrm{C}, 100 \% \mathrm{GF}$ in D) for 7 days exhibit differentiation into astrocytes (stained by antibody against GFAP, green). Phase contrast images (A) and fluorescence micrographs (B, C, D) showing stained nuclei (Hoechst, blue) identify all cells in the field. Note the significantly higher proportion of astrocytes in the low GF compartment compared to the other two compartments (B).

stained with antibodies to astrocytes, one of the main cell types that differentiate from hNSCs. Cells in the low GF compartment showed increased astrocyte differentiation compared to cells in the high GF compartment (Fig. 5B). This pattern was similar to that of cells on coverslips in parallel conventional culture experiments (Fig. 5C, D). Importantly, the percentage of astrocytes across the three GF compartments in the gradient chamber decreased with increasing GF concentration (Fig. 6). Astrocyte percentages in the gradient chamber were consistent with those seen in the control microfluidic chambers and on the coverslips in conventional cultures. We further analyzed the data after subdividing each compartment into 8 equal bins, as done to analyze total cell numbers. We continued to find strong correlations between astrocyte percentage and GF concentration in all three compartments (Fig. 6C and data not shown; ESI Table $\mathrm{S} 2 \uparrow$ for $p$ values). Thus, astrocyte differentiation was inversely proportional to GF concentration, while proliferation was directly proportional. Like the proliferation effect, no threshold response in astrocyte differentiation was seen at the population level over the GF concentrations used in these experiments.

\section{Conclusions}

These results show that hNSCs can be cultured in a microfluidic device under constant fluid flow for more than 1 week using minimal reagents $(\sim 1 \mathrm{~mL}$ media per syringe $)$ and without any detectable detrimental effects on cell adhesion, proliferation, or differentiation. By applying a continuous gradient of known GFs across the cells, opposing effects on proliferation and astrocyte differentiation could be induced, both of which were graded and proportional to GF
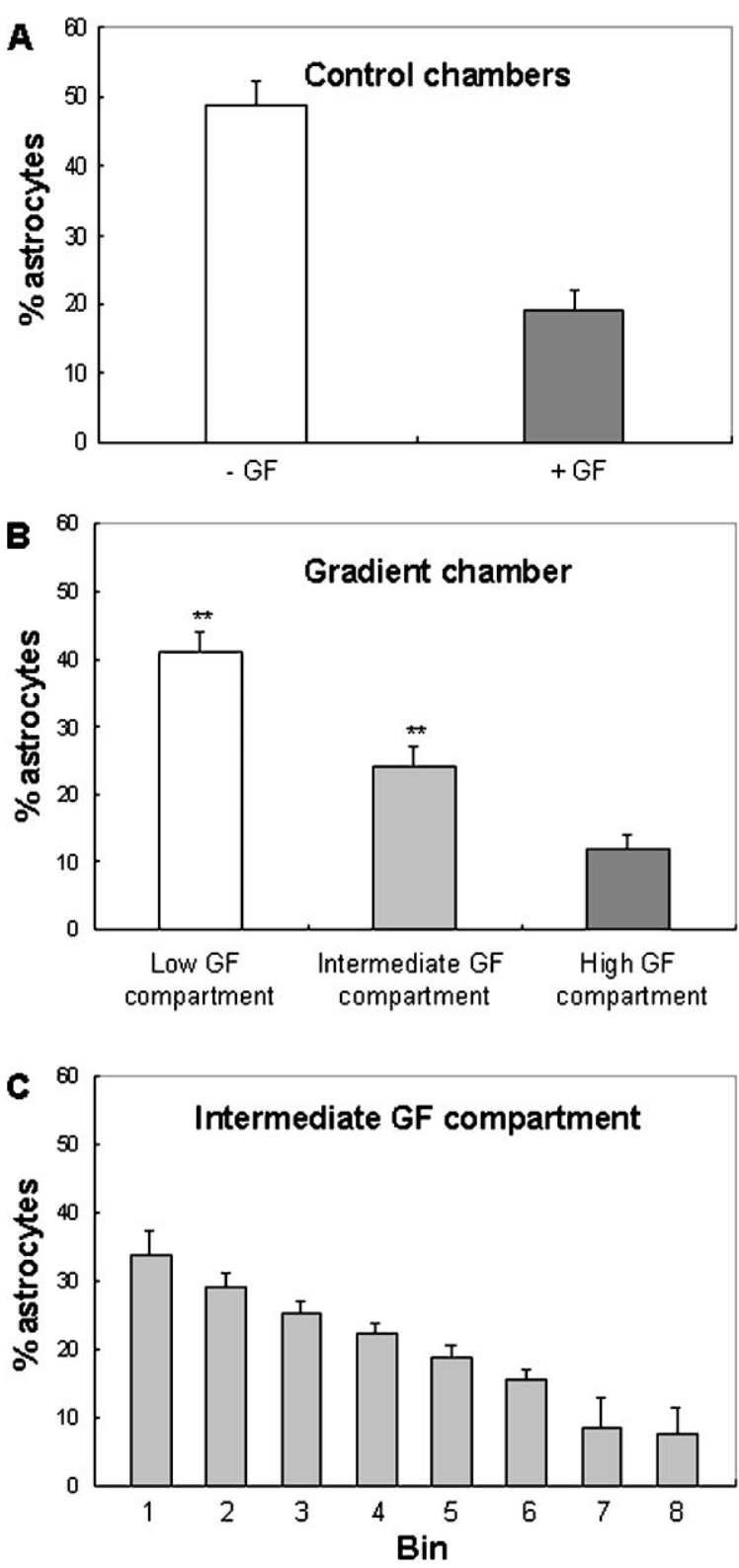

Fig. 6 Quantitation of astrocytes in the control (A) and gradient chambers (B, C) after 7 days. Astrocytes are expressed as a percentage of the total number of Hoechst-stained cells. Note that astrocyte percentage is inversely proportional to GF concentration in the gradient chamber. Each bar indicates the average for 3 independent experiments with standard errors of the mean $\left({ }^{* *} p<0.01\right)$. The strong negative correlation between astrocyte percentage and GF concentration persists after subdividing the intermediate GF compartment into 8 bins (C; ESI Table $\mathrm{S} 2 \uparrow$ for $p$ values).

concentration. Neither threshold effects nor plateaus in proliferation or differentiation responses were detected at the population level in the GF concentration ranges used. Rather than requiring multiple independent traditional cultures using fixed GF concentrations, the microfluidic culture device allowed these graded responses to be induced and quantified within a single culture chamber. Additional advantages of this microfluidic platform over traditional cultures - such as the minimization of autocrine and paracrine signals, the ability to 
apply multiple gradients simultaneously, the ability to micropattern the cell culture surface area, and the low media and cell requirements - should enable the optimization and control of hNSC and other SCs in culture prior to use in transplantation and tissue engineering protocols.

\section{Acknowledgements}

This paper was supported by seed grants from UCI CORCLR, UCI College of Medicine/Biomedical Engineering, and a Roman Reed Research Award from the State of California.

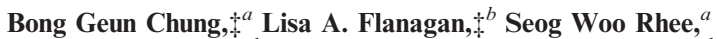

Philip H. Schwartz, ${ }^{c d}$ Abraham P. Lee, ${ }^{a}$ Edwin S. Monuki ${ }^{* b c}$ and Noo Li Jeon*a

${ }^{a}$ Department of Biomedical Engineering, Henry Samueli School of Engineering, University of California Irvine, Irvine, CA 92697-2715, USA. E-mail: njeon@uci.edu; Fax: 1-949-824-9968;

Tel: 1-949-824-9032

${ }^{b}$ Department of Pathology, College of Medicine, University of California Irvine, Irvine, CA 92697-4800, USA. E-mail: emonuki@uci.edu;

Fax: 1-949-824-2160; Tel: 1-949-824-9604

${ }^{c}$ Department of Developmental and Cell Biology, School of Biological Sciences, University of California Irvine, Irvine, CA, USA

${ }^{d}$ National Human Neural Stem Cell Resource, Children's Hospital of

Orange County Research Institute, Orange, CA, USA

\section{References}

1 P. H. Schwartz, P. J. Bryant, T. J. Fuja, H. Su, D. K. O’Dowd and H. Klassen, J. Neurosci. Res., 2003, 74, 838-851.

2 T. D. Palmer, P. H. Schwartz, P. Taupin, B. Kaspar, S. A. Stein and F. H. Gage, Nature, 2001, 411, 42-43.

3 H. S. Keirstead, J. Neurosci. Res., 2001, 63, 233-236.

4 C. N. Svendsen and A. G. Smith, Trends. Neurosci., 1999, 22, 357-364.

5 P. W. Zandstra and A. Nagy, Annu. Rev. Biomed. Eng., 2001, 3, 275-305.

6 D. J. Anderson, Neuron, 2001, 30, 1, 19-35.

7 B. A. Reynolds, W. Tetzlaff and S. Weiss, J. Neurosci., 1992, 12, $11,4565-4574$.

8 C. T. Gregg, A. K. Chojnacki and S. Weiss, J. Neurosci. Res., 2002, 69, 708-713.

9 N. L. Kennea and H. Mehmet, J. Pathol, 2002, 197, 536-550.

10 F. H. Gage, Science, 2000, 287, 25, 1433-1438.
11 S. Temple, Nature, 2001, 414, 1, 112-117.

12 D. J. Beebe, G. A. Mensing and G. M. Walker, Annu. Rev. Biomed. Eng., 2002, 4, 261-286.

13 S. K. Sia and G. M. Whitesides, Electrophoresis, 2003, 24, 3563-3576.

14 J. M. K. Ng, I. Gitlin, D. stroock Abraham and G. M. Whitesides, Electrophoresis, 2002, 23, 3461-473.

15 N. L. Jeon, H. Baskaran, S. K. W. Dertinger, G. M. Whitesides, L. V. D. Water and M. Toner, Nat. Biotechnol., 2002, 20, 826-830.

16 S. J. Wang, W. Saadi, F. Lin, C. M. Nguyen and N. L. Jeon, Exp. Cell. Res., 2004, 300, 1, 180-189.

17 W. Gu, X. Zhu, N. Futai, B. S. Cho and S. Takayama, Proc. Natl. Acad. Sci, 2004, 101, 15861-15866.

18 S. Raty, E. M. Walters, J. Davis, H. Zeringue, D. J. Beebe, S. L. Rodriguez-Zas and M. B. Wheeler, Lab Chip, 2004, 4, 186-190.

19 A. Tourovskaia, X. Rodriguez-Zas and A. Folch, Lab Chip, 2005, 5, 1, 14-19.

20 S. Takayama, J. C. McDonald, E. Ostuni, M. N. Liang, P. J. A. Keins, R. F. Ismagilov and G. M. Whitesides, Proc. Natl. Acad. Sci., 1999, 96, 5545-5548.

21 N. L. Jeon, S. K. W. Dertinger, D. T. Chiu and G. M. Whitesides, Langmuir, 2000, 16, 8311-8316.

22 S. K. W. Dertinger, D. T. Chiu, N. L. Jeon and G. M. Whitesides, Anal. Chem., 2001, 73, 1240-1246.

23 D. C. Duffy, J. C. McDonald, O. J. A. Schueller and G. M. Whitesides, Anal. Chem., 1998, 70, 4974- 4984.

24 D. Qin, Y. Xia and G. M. Whitesides, Adv. Mater., 1996, 8, 917-921.

25 Y. Xia and G. M. Whitesides, Angew. Chem., Int. Ed. Engl., 1998, 37, 550-575.

26 G. M. Whitesides, E. Ostuni, S. Takayama, X. Jiang and D. E. Ingber, Annu. Rev. Biomed. Eng., 2001, 3, 335-373.

27 T. Ostenfeld and C. N. Svendsen, Stem Cells, 2004, 22, 5, 798-811.

28 A. L. Vescovi, E. A. Parati, A. Gritti, P. Poulin, M. Ferrario, E. Wanke, P. Frolichsthal-Schoeller, L. Cova, M. ArcellanaPanlilio, A. Colombo and R. Galli, Exp. Neurol., 1999, 156, 71-83.

29 G. M. Wheeler, H. C. Zeringue and D. J. Beebe, Lab Chip, 2004, 4, 2, 91-97.

30 S. W. Rhee, A. M. Taylor, C. H. Tu, D. H. Cribbs, C. W. Cotman and N. L. Jeon, Lab Chip, 2005, 5, 1, 102-107.

31 G. Banker and K. Golsin, Culturing Nerve Cells, The MIT Press, 2nd edn., Cambridge, 1998, ch. 13.

32 F. Lin, W. Saadi, S. W. Rhee, S. Wang, S. Mittal and N. L. Jeon, Lab Chip, 2004, 4, 164-167.

33 R. P. Franke, M. Grafe, H. Schnittler, D. Seiffge and C. Mittermayer, Nature, 1984, 307, 16, 648-649. 